\title{
Elaboração e Validação de Escala Diagramática para Avaliação da Severidade da Mancha Preta em Frutos Cítricos*
}

\author{
Marcel B. Spósito ${ }^{1 * *}$, Lilian Amorim ${ }^{2 * *}$, José Belasque Junior ${ }^{1 * *}$, Renato B. Bassanezi ${ }^{1}$ \& Renata de \\ Aquino ${ }^{1 * *}$
}

${ }^{1}$ Departamento Científico, Fundecitrus, Cx. Postal 391, CEP 14801-970, Araraquara, SP, fax: (16) 201-7032, e-mail: marcel@ fundecitrus.com.br; ${ }^{2}$ Departamento de Entomologia, Fitopatologia e Zoologia Agrícola, ESALQ-USP, Cx. Postal 9, CEP 13418-900, Piracicaba, SP, e-mail: liamorim@esalq.usp.br

(Aceito para publicação em 02/10/2003)

Autor para correspondência: Marcel B. Spósito

SPÓSITO, M.B., AMORIM, L., BELASQUE JUNIOR, J., BASSANEZI, R.B. \&. AQUINO, R. Elaboração e validação de escala diagramática para avaliação da severidade da mancha preta em frutos cítricos. Fitopatologia Brasileira 29:081-085. 2004.

\section{RESUMO}

Uma escala diagramática, abrangendo os dois tipos prevalentes de sintomas de mancha preta em frutos cítricos (Citrus spp.), os de mancha dura e de falsa melanose, foi desenvolvida para padronizar a avaliação da severidade da doença. A escala foi elaborada considerando os limites máximos e mínimos de severidade da doença observados no campo. Os valores intermediários seguiram incrementos logarítmicos para os sintomas do tipo mancha dura $(0,5 ; 1,7 ; 5,0 ; 11,5 ; 22,5$ e $49,0 \%)$ e do tipo falsa melanose $(1,1 ; 4,5 ; 15,0 ; 31,0 ; 53,0$ e $68,0 \%)$. Para a validação da escala, seis avaliadores quantificaram a severidade da doença a partir das imagens digitalizadas de 50 frutos com diferentes níveis de doença. Inicialmente, a estimativa da severidade foi feita sem auxílio da escala. Em seguida, os mesmos avaliadores, utilizando a escala diagramática proposta, estimaram a severidade nos mesmos frutos avaliados anteriormente. As avaliações com a escala diagramática foram mais precisas e acuradas nas estimativas de todos os avaliadores e proporcionaram maior reprodutibilidade entre avaliações de diferentes avaliadores. A escala diagramática proposta foi considerada adequada para estimar a severidade da mancha preta nos frutos e será usada em estudos epidemiológicos e de avaliação de estratégias de controle desta doença.

Palavras-chave adicionais: Citrus sinensis, pinta preta dos citros, Guignardia citricarpa, patometria.

\section{ABSTRACT}

Elaboration and validation of diagrammatic scale to evaluate black spot severity in citrus fruits

A diagrammatic scale, showing the most common symptoms of black spot in citrus (Citrus spp.) fruits, hard spot and false melanose types, was developed to standardize severity assessment. The scale was elaborated considering the maximum and minimum limits of disease severity observed in the field. The intermediate values followed logarithmic increments for hard spot $(0.5,1.7,5.0$, $11.5,22.5$, and $49.0 \%)$ and false melanose $(1.1,4.5,15.0,31.0,53.0$, and $68.0 \%$ ) symptoms. To validate the scale, six raters quantified the disease severity of digitalized images of 50 fruits with black spot symptoms. Initially, the estimates of severity were done without the use of the scale. Then, the raters estimated the severity of the same fruits previously assessed using the proposed diagrammatic scale. Assessments with the diagrammatic scale were of greater precision and higher accuracy for all raters, besides being more highly reproducible among the different raters. The proposed diagrammatic scale was considered adequate to estimate black spot severity in citrus fruits and will be used in epidemiological studies and int he evaluation of control strategies for this disease.

\section{INTRODUÇÃO}

A mancha preta dos citros (Citrus spp.), incitada pelo fungo Guignardia citricarpa Kiely, é doença de importância econômica, principalmente para a região sul do Estado de São Paulo (Feichtenberger et al., 1997). O patógeno causa lesões em frutos, ocasionando queda precoce, podendo reduzir a produção em até $80 \%$ (Klotz, 1978). Os frutos com sintomas também são depreciados para a comercialização no mercado de fruta fresca, resultando menor rentabilidade para o citricultor

\footnotetext{
* Parte da Tese de Doutorado do primeiro autor. Escola Superior de Agricultura "Luiz de Queiroz", USP, parcialmente financiada pela FAPESP (01/05508-5).

** Bolsita CNPq.
}

que atende este setor.

A doença manifesta-se de diversas formas nos frutos, sendo relatados, até o momento, seis tipos de sintomas diferentes (Aguilar-Vildoso et al., 2002). Os sintomas mais comuns observados em frutos são os do tipo mancha dura e os da falsa melanose (Kotzé, 1981; 1988). O sintoma do tipo mancha dura caracteriza-se por lesões circulares, deprimidas, com bordos salientes de coloração marrom e na maioria das vezes por apresentar pontuações negras no seu interior, que correspondem aos picnídios. Este tipo de sintoma normalmente ocorre no período de mudança da coloração dos frutos. O sintoma do tipo falsa melanose caracteriza-se por minúsculas e numerosas pontuações escuras, dispersas ou agregadas, que normalmente aparecem em frutos ainda verdes (Feichtenberger et al., 1997). 
A quantificação de doenças é fundamental para estudos epidemiológicos e para avaliação de estratégias de controle. A quantificação de uma variável que expresse a intensidade de doença, quer seja ela medida pela incidência ou pela severidade, é necessária, tanto para descrever o progresso da epidemia e sua relação com o clima ou com medidas de controle, quanto para validação de modelos de previsão ou para a aplicação do manejo integrado. Portanto, erros na quantificação da doença podem ser magnificados na análise epidemiológica subseqüente, interferindo em maior ou menor grau, nas conclusões alcançadas (Campbell \& Madden, 1990). Para que os erros sejam minimizados, o método utilizado na quantificação da doença deve ser capaz de fornecer resultados acurados, precisos e reproduzíveis.

A avaliação da mancha preta dos citros tem sido realizada pela incidência da doença, quando os frutos são destinados ao mercado de fruta fresca, ou pela severidade da doença, quando os frutos são destinados à produção de suco concentrado. A quantificação da severidade é comumente realizada com o auxílio de escalas diagramáticas representando frutos com diferentes porcentagens de área ocupadas pelas lesões. As escalas são utilizadas na orientação da estimativa visual de tal modo que a avaliação torne-se mais precisa e acurada após a comparação da amostra com a representação no diagrama. Quando a avaliação visual é feita sem o auxílio de escalas diagramáticas, o avaliador pode atribuir qualquer valor no intervalo de zero a $100 \%$ para a superfície do fruto ocupada por sintomas. $\mathrm{O}$ uso da escala permite ao avaliador situar sua estimativa num intervalo menor, compreendido entre dois dos níveis representados por seus diagramas (Amorim, 1995). Dessa forma, as escalas diagramáticas contribuem na redução do erro na estimativa visual da severidade de doenças.

As escalas diagramáticas devem ser simples, aplicáveis em diferentes condições e ter intervalos suficientes para representar os diferentes níveis de severidade de uma determinada doença. Na elaboração de uma escala diagramática, alguns aspectos devem ser considerados, como as quantidades mínima e máxima de doença observadas no campo e representadas na escala, a representação dos sintomas da maneira mais próxima possível da realidade e os níveis de severidade respeitando as limitações da acuidade da vista humana, definidas pelas leis de estímulo-resposta de Weber e de Fechner, também conhecidas como lei de Weber-Fechner (Horsfall \& Barrat, 1945; Nutter \& Schultz, 1995). Além da boa qualidade de uma escala diagramática, as estimativas de severidade dependem da percepção visual e da experiência de cada indivíduo na avaliação de doenças.

A precisão e a acurácia das estimativas de severidade variam de acordo com o avaliador. Após a elaboração, as escalas devem ser testadas por diferentes indivíduos a fim de comprovar sua eficiência na estimativa da severidade. A precisão, medida de confiabilidade e/ou repetibilidade nas avaliações de doença (Nutter et al., 1991), pode ser quantificada pelo coeficiente de determinação de regressões lineares estabelecidas entre a severidade real e as estimativas da severidade de uma determinada amostra. A precisão será tanto maior quanto mais próximo de 1,0 for o valor do coeficiente de determinação $\left(R^{2}\right)$. A acurácia, representada pelo grau de proximidade entre a estimativa média e a realidade (Nutter $e t$ al., 1991), pode ser quantificada pelo coeficiente angular e pela interseção de linhas de regressão estabelecidas entre a severidade real e as estimativas da severidade de uma determinada amostra. A acurácia será tanto maior quanto mais próximo de 1,0 for o coeficiente angular e mais próximo de zero for a interseção.

Aguilar-Vildoso et al. (2002) publicaram uma escala diagramática para a avaliação da mancha preta dos citros, a qual vem sendo utilizada, principalmente, em ensaios de comparação de tratamentos para o controle da doença. No entanto, essa escala não atende às necessidades de quantificação para estudos epidemiológicos, pois a severidade máxima representada na escala é de $25 \%$, muito abaixo da severidade observada no campo na ausência de medidas de controle. Além disso, apenas sintomas do tipo mancha dura estão representados nessa escala, dificultando a avaliação de frutos com sintomas do tipo falsa melanose.

O objetivo deste trabalho foi elaborar e validar uma nova escala diagramática para avaliação da mancha preta dos citros na qual estivessem representados tanto sintomas do tipo mancha dura quanto sintomas do tipo falsa melanose.

\section{MATERIALEMÉTODOS}

Para a quantificação dos níveis representados na escala diagramática, 50 frutos de laranja doce (Citrus sinensis Osbeck) das variedades 'Hamlin', 'Pera', 'Valência' e 'Natal' com ampla variação de severidade da doença $(0,5 \%$ a $68 \%)$, foram coletados em fazendas de citros na região de Mogi Guaçu, em talhões com $100 \%$ de incidência da doença. A face externa de cada fruto, levando em consideração o seu posicionamento na planta, foi fotografada com câmera digital C-2020 Zoom Digital Camera (Olympus $\left.{ }^{\circledR}\right)$. As imagens digitalizadas foram transferidas para um microcomputador e, posteriormente, foram processadas com o programa SIARCS 3.0, elaborado e comercializado pelo Centro Nacional de Pesquisa e Desenvolvimento de Instrumentação Agropecuária da Embrapa (Jorge, 1996). Tanto a área necrosada como o halo amarelado, eventualmente presente, foram considerados como tecido doente para fins de quantificação da severidade. Os níveis mínimo e máximo de severidade foram determinados de acordo com a maior frequiência observada nos frutos coletados. Os níveis intermediários da escala foram determinados matematicamente, seguindo incrementos logarítmicos. Depois de estabelecidas as porcentagens de doença a serem representadas na escala, reproduziu-se um desenho padrão com área conhecida, representando um fruto cítrico, ao qual acrescentaram-se desenhos de lesões de formatos variados, distribuídos de maneira a retratar os sintomas observados no campo. Foram representados seis níveis de doença com sintomas do tipo mancha dura e seis níveis de doença com sintomas do tipo falsa melanose.

Para a validação da escala, seis avaliadores quantificaram a severidade da doença a partir das imagens dos 50 
Elaboração e validação de escala diagramática para avaliação da...

frutos, apresentadas com auxílio de um projetor tipo "multimedia", em duas fases. Em uma primeira fase, os avaliadores estimaram a severidade sem auxílio da escala diagramática e, em uma segunda fase, estimaram a severidade com o auxílio da mesma. Quando a escala diagramática foi utilizada, a opção pela escolha da representação dos sintomas do tipo mancha dura ou da representação dos sintomas do tipo falsa melanose ficou a critério de cada avaliador. Essa informação não foi monitorada.

Regressões lineares entre a severidade real (variável independente) e a estimada (variável dependente) foram calculadas de acordo com a metodologia de Draper \& Smith (1998) e Teng (1981), para cada avaliador, individualmente, e conjuntamente para todos os avaliadores. A acurácia das estimativas de cada avaliador e do conjunto de avaliadores foi determinada pelo teste $t$ aplicado às estimativas dos parâmetros interseção da regressão linear $(a)$, para verificar a hipótese Ho: $a=0$, e coeficiente angular da reta $(b)$, para testar a hipótese Ho: $b=1$, ao nível de probabilidade $p=0,05$. A precisão foi estimada pelo coeficiente de determinação da regressão $\left(R^{2}\right)$ e pela variação dos resíduos (severidade estimada menos a severidade real) (Kranz, 1988; Campbell \& Madden, 1990; Nutter Jr. \& Schultz, 1995). A comparação entre os parâmetros $a$ e $b$ das estimativas sem $\left(a_{s}\right.$ e $\left.b_{s}\right)$ e com $\left(a_{c}\right.$ e $\left.b_{c}\right)$ auxílio da escala diagramática ao conjunto dos dados de todos os avaliadores foi realizada pelo teste $t$, para verificar as hipóteses Ho: $a_{s}=a_{c}$ e Ho: $b_{s}=b_{c}$, ao nível de probabilidade de $p=0,05$ (Campbell \& Madden, 1990). A reprodutibilidade das avaliações entre os diferentes avaliadores foi testada por meio de regressões lineares onde tanto a variável dependente quanto a independente foram estimativas de diferentes avaliadores. Todos os avaliadores foram confrontados entre si. Avaliou-se a precisão e acurácia das estimativas de todas as combinações. O programa Excel 97 $\left(\right.$ Microsoft $\left.^{\circledR}\right)$ foi utilizado nas análises de regressão linear.

\section{RESULTADOS E DISCUSSÃO}

A escala diagramática para mancha preta dos citros foi desenhada com seis níveis de severidade para os sintomas do tipo mancha dura $(0,5 ; 1,7 ; 5,0 ; 11,5 ; 22,5$ e 49,0\%) e seis níveis de severidade para os sintomas do tipo falsa melanose $(1,1$; 4,5; 15,0; 31,0; 53,0 e 68,0\%) (Figura 1).

As avaliações de severidade da mancha preta dos citros, feitas sem e com o auxílio da escala, levaram em consideração somente a face externa de cada fruto fotografado. No campo, rotineiramente avalia-se a área dos frutos mais expostas aos raios solares, pois é neste local que há maior expressão dos sintomas da doença (Kotzé, 1981; 1988).

Quando a severidade da doença foi estimada sem o auxílio da escala diagramática, os avaliadores foram relativamente precisos, com coeficientes de determinação variando entre 0,85 e 0,91 para as retas de regressão entre a severidade real e a estimada (Tabela 1). Entretanto, os seis avaliadores superestimaram a severidade da doença, incorrendo em erros sistemáticos nas estimativas (Figura 2A-F). A interseção da reta $(a)$ foi sempre elevada, com valor médio,

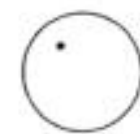

0.5

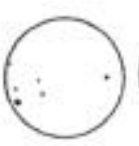

1.1

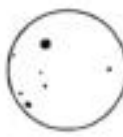

1.7

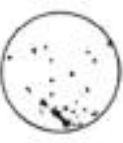

4,5

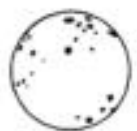

5.0

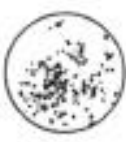

15.0

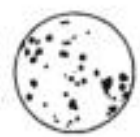

11.5

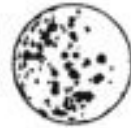

22.5

49.0

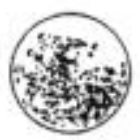

31,0

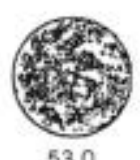

53.0

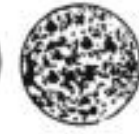

FIG. 1 - Escala diagramática para avaliação da severidade (\%) da mancha preta (Guignardia citricarpa) dos citros (Citrus spp.). Os diagramas da parte superior são representações do sintoma de mancha dura e os da parte inferior, representações do sintoma de falsa melanose.

obtido pela regressão conjunta de todos avaliadores, de 5,26 (Figura 2G). Todos os valores de $a$ foram significativamente diferentes de zero (Tabela 1). O coeficiente angular da reta de regressão $(b)$ também foi elevado, com um valor médio de 1,26, estatisticamente diferente de 1,0 (Tabela 1). Os valores de $b$ foram estatisticamente diferentes de 1,0 para quatro dos seis avaliadores (Tabela 1). A distribuição dos resíduos das avaliações realizadas sem o auxílio da escala diagramática apresentou estimativas com erros absolutos variando entre 0,38 (Figura 3F) e 44,70 (Figura 3A), sendo a média dos erros (em módulo) de todos os avaliadores igual a 7,26.

Quando a avaliação foi realizada com o auxílio da escala, todos os avaliadores melhoraram sua precisão, com coeficientes de determinação variando entre 0,89 e 0,96 (Tabela 1), e reduziram os erros sistemáticos das estimativas (Figura $2 \mathrm{H}-\mathrm{M})$. O valor médio da interseção da regressão $(a)$ foi de 2,35 (Tabela 1). O uso da escala resultou em coeficientes angulares próximos de 1,0 para quatro avaliadores e em três

TABELA 1 - Estimativas de parâmetros e coeficientes de determinação $\left(R^{2}\right)$ das equações de regressão linear calculadas entre severidade real e severidade estimada de mancha preta dos citros (Citrus spp.) realizadas por seis avaliadores individualmente e para o conjunto dos mesmos, sem e com o uso de escala diagramática $(y=a+b x$, em que $y$ representa a severidade estimada e $x$, a severidade real)

\begin{tabular}{|c|c|c|c|c|c|c|}
\hline \multirow{2}{*}{ Avaliador } & \multicolumn{3}{|c|}{ Sem escala } & \multicolumn{3}{|c|}{ Com escala } \\
\hline & $a^{(\mathbf{i})}$ & $b^{(\text {ii) }}$ & $R^{2}$ & $a$ & $b$ & $R^{2}$ \\
\hline 1 & $8,64 *$ & $1,74 *$ & 0,91 & $2,16^{*}$ & $0,98 \mathrm{~ns}$ & 0,94 \\
\hline 2 & $5,29 *$ & $1,11 \mathrm{~ns}$ & 0,88 & $1,94 *$ & $1,13 *$ & 0,95 \\
\hline 3 & $6,29 *$ & $0,98 \mathrm{~ns}$ & 0,89 & $1,80 *$ & $0,90 *$ & 0,95 \\
\hline 4 & $5,17 *$ & $1,22 *$ & 0,85 & $3,65 *$ & $0,96 \mathrm{~ns}$ & 0,89 \\
\hline 5 & $4,07 *$ & $1,11 *$ & 0,89 & $3,21 *$ & $0,95 \mathrm{~ns}$ & 0,92 \\
\hline 6 & $2,09 *$ & $1,39 *$ & 0,91 & $1,29 *$ & $1,21 *$ & 0,96 \\
\hline Todos & $5,26 *$ & $1,26 *$ & 0,83 & $2,35 *$ & $1,02 \mathrm{~ns}$ & 0,92 \\
\hline
\end{tabular}

(i)asterisco indica que o valor da interseção da reta $(a)$ foi diferente de zero pelo teste $t(p=0,05)$, ns indica que não houve diferença estatística significativa entre $a$ e zero pelo teste $t(p=0,05)$;

(ii)asteriscos indicam que o valor do coeficiente angular da reta de regressão (b) foi diferente de um pelo teste $t(p=0,05)$, ns indica que não houve diferença estatística significativa entre $b$ e um pelo teste $t(p=0,05)$. 
M.B. Spósito et al.
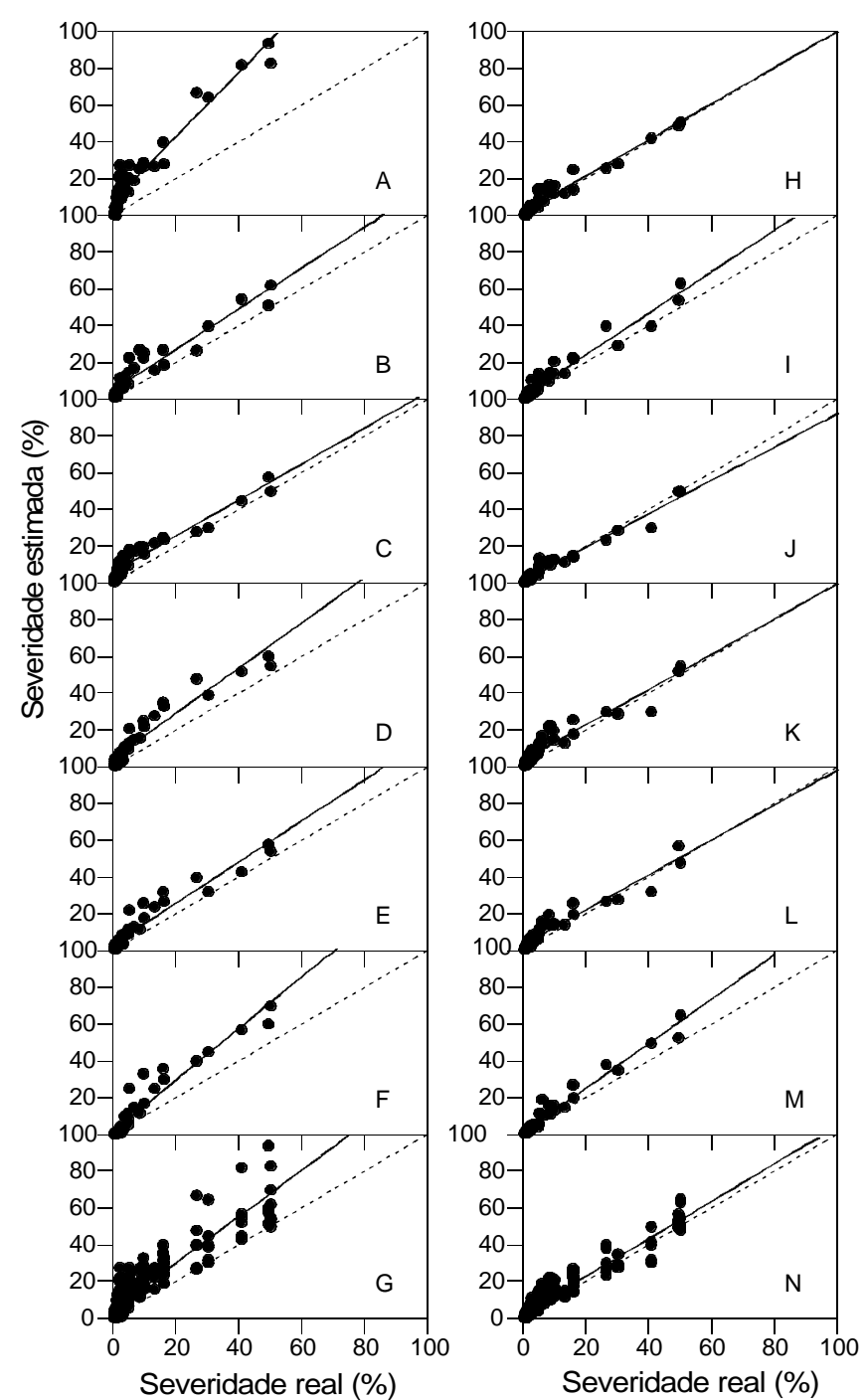

FIG. 2 - Severidade estimada $(\bullet)$ sem auxílio (A-G) e com o auxílio da escala diagramática para mancha preta $(\mathrm{H}-\mathrm{N})$ dos citros (Citrus spp.), por seis avaliadores: avaliador $1(\mathrm{~A}, \mathrm{H}), 2$ (B, I), 3 (C, J), 4 (D, K), 5 (E, L) e $6(\mathrm{~F}, \mathrm{M})$, e pelo conjunto dos mesmos $(\mathrm{G}, \mathrm{N})$. As linhas cheias representam a regressão linear entre a severidade real e a estimada. Linhas tracejadas representam a situação ideal em que a severidade estimada é igual à real.

deles (1, 4 e 5) não diferiram estatisticamente de 1,0 (Tabela 1$)$. $\mathrm{O}$ valor do coeficiente angular da reta $(b)$ de 1,02 , obtido na regressão conjunta de todos os avaliadores com o uso de escala (Figura 2N), foi estatisticamente igual a 1,0 (Tabela 1). Os valores dos parâmetros $a$ e $b$ obtidos na avaliação com o auxílio da escala foram significativamente menores que os obtidos sem a escala, para o conjunto dos seis avaliadores. Houve redução nos erros absolutos para as estimativas com auxílio da escala, quando comparada com a distribuição dos resíduos das estimativas obtidas sem escala diagramática. Os erros absolutos variaram entre -2,89 (Figura 3I) e 15,03 (Figura $3 \mathrm{~L}$ ), sendo a média dos erros (em módulo) de todos avaliadores igual 2,87. A maioria dos erros absolutos observados para as estimativas obtidas por todos os avaliadores, com o auxílio da
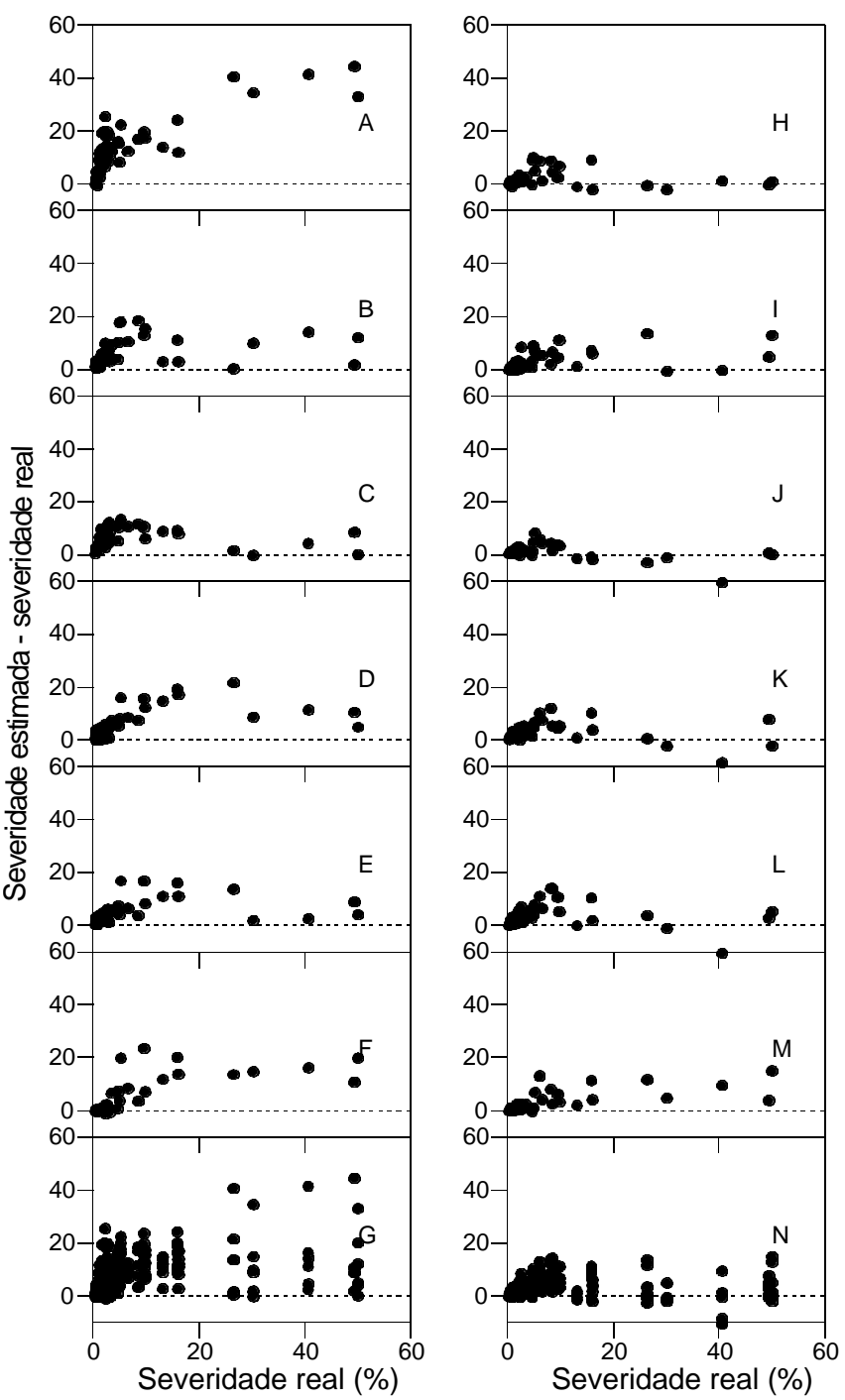

FIG. 3 - Resíduos $(\bullet)$ das análises de regressão linear entre severidade real e severidades estimadas sem (A-G) e com (H-N) o auxílio de escala diagramática para mancha preta dos citros (Citrus spp.), por seis avaliadores e pelo conjunto dos mesmos: avaliador $1(\mathrm{~A}, \mathrm{H}), 2$ (B, I), $3(\mathrm{C}, \mathrm{J}), 4(\mathrm{D}, \mathrm{K}), 5$ (E, L) e $6(\mathrm{~F}, \mathrm{M})$ e para o conjunto (G, N).

escala, ficou dentro de valores aceitáveis $(-10$ a $+10 \%)$, segundo critérios adotados por programas de treinamento na quantificação de doenças, como o Distrain (Tomerlin \& Howell, 1988) e o Disease.Pro (Nutter \& Worawitlikit, 1989).

Os coeficientes de determinação das equações de regressão linear calculadas nas comparações das estimativas dos avaliadores entre si foram maiores quando estes utilizaram a escala diagramática do que sem a utilização desta (Tabela 2). Entretanto, não houve diferença quanto às estimativas dos parâmetros $a$ e $b$ entre os avaliadores quando estes utilizaram a escala ou não (Tabela 2). Os avaliadores, quando utilizaram a escala, foram mais precisos entre si na estimativa da severidade da doença. Do ponto de vista prático, o uso da escala diagramática aumenta a reprodutibilidade das estimativas obtidas por diferentes avaliadores e, portanto, a estimativa da severidade da doença poderá ser feita por qualquer avaliador, pois os 
Elaboração e validação de escala diagramática para avaliação da...

TABELA 2 - Estimativas de parâmetros e coeficientes de determinação $\left(R^{2}\right)$ das equações de regressão linear calculadas entre as severidades de mancha preta dos citros (Citrus spp.) estimadas por diferentes avaliadores $(y=a+b x$, em que $y$ representa a severidade estimada por um avaliador e $x$, a severidade estimada por outro avaliador). As estimativas da severidade dos seis avaliadores foram confrontadas entre si, sem e com o uso de escala diagramática.

\begin{tabular}{|c|c|c|c|c|c|c|}
\hline \multirow{2}{*}{$\begin{array}{l}\text { Comparação } \\
\text { entre } \\
\text { avaliadores }\end{array}$} & \multicolumn{3}{|c|}{ Sem escala } & \multicolumn{3}{|c|}{ Com escala } \\
\hline & $a^{(i)}$ & $b^{(\text {ii) }}$ & $R^{2}$ & $a$ & $b$ & $R^{2}$ \\
\hline $1 \times 2$ & $0,52 \mathrm{~ns}$ & $0,60 *$ & 0,87 & $-0,20 \mathrm{~ns}$ & $1,11 *$ & 0,94 \\
\hline $1 \times 3$ & $1,98 \mathrm{~ns}$ & $0,54 *$ & 0,89 & $0,09 \mathrm{~ns}$ & $0,89 *$ & 0,95 \\
\hline $1 \times 4$ & $-0,09 n s$ & $0,66 *$ & 0,83 & $1,60 \mathrm{~ns}$ & $0,97 \mathrm{~ns}$ & 0,93 \\
\hline $1 \times 5$ & $-0,65 \mathrm{~ns}$ & $0,60 *$ & 0,87 & $1,25 \mathrm{~ns}$ & $0,95 \mathrm{~ns}$ & 0,95 \\
\hline $1 \times 6$ & $-3,67 *$ & $0,75^{*}$ & 0,86 & $-0,98 \mathrm{~ns}$ & $1,19 *$ & 0,95 \\
\hline $2 \times 3$ & $2,34 \mathrm{~ns}$ & $0,83 \mathrm{~ns}$ & 0,89 & $0,47 \mathrm{~ns}$ & $0,78 *$ & 0,95 \\
\hline $2 \times 4$ & $0,44 \mathrm{~ns}$ & $1,02 \mathrm{~ns}$ & 0,82 & $2,05 *$ & $0,85 *$ & 0,93 \\
\hline $2 \times 5$ & $-0,16 \mathrm{~ns}$ & $0,92 \mathrm{~ns}$ & 0,85 & $1,78 *$ & $0,82 *$ & 0,93 \\
\hline $2 \times 6$ & $-3,49 *$ & $1,18 *$ & 0,89 & $-0,44 \mathrm{~ns}$ & $1,03 \mathrm{~ns}$ & 0,95 \\
\hline $3 \times 4$ & $-2,13 n s$ & $1,21 *$ & 0,89 & $1,69 *$ & $1,07 \mathrm{~ns}$ & 0,94 \\
\hline $3 \times 5$ & $-2,39 *$ & $1,09 \mathrm{~ns}$ & 0,91 & $1,34 *$ & $1,05 \mathrm{~ns}$ & 0,96 \\
\hline $3 \times 6$ & $-5,78 *$ & $1,34 *$ & 0,89 & $-0,76 n s$ & $1,29 *$ & 0,95 \\
\hline $4 \times 5$ & $-0,09 n s$ & $0,87 *$ & 0,96 & $-0,03 n s$ & $0,95 \mathrm{~ns}$ & 0,96 \\
\hline $4 \times 6$ & $-2,84 *$ & $1,07 \mathrm{~ns}$ & 0,94 & $-2,29 *$ & $1,16 *$ & 0,93 \\
\hline $5 \times 6$ & $-2,67 *$ & $1,23 *$ & 0,97 & $-2,08 *$ & $1,20 *$ & 0,93 \\
\hline
\end{tabular}

(i)asterisco indica que o valor da interseção da reta $(a)$ foi diferente de zero pelo teste $t(p=0,05)$, ns indica que não houve diferença estatística significativa entre $a$ e zero pelo teste $t(p=0,05)$;

(ii)asteriscos indicam que o valor do coeficiente angular da reta de regressão (b) foi diferente de um pelo teste $t(p=0,05)$, ns indica que não houve diferença estatística significativa entre $b$ e um pelo teste $t(p=0,05)$.

resultados serão semelhantes e diferentes experimentos, conduzidos por avaliadores distintos, serão comparáveis.

A média geral das estimativas de severidade da doença de todos os avaliadores ficou em 14,6 quando estes não utilizaram a escala e 9,9, quando utilizaram a escala diagramática. A média geral dos valores reais de severidade dos frutos fotografados foi de 7,4. Portanto, a utilização da escala fez com que os avaliadores estimassem a severidade com maior precisão. $\mathrm{O}$ desvio padrão dos valores estimados por todos avaliadores quando não utilizaram a escala foi de 3,45. Após a utilização da escala esse valor foi de 0,82 . Essa menor variabilidade das estimativas com o uso da escala reforça a reprodutibilidade das estimativas de severidade da doença quando utilizou-se a escala diagramática. Isso corrobora com a opinião de Nutter $e t$ al. (1998), que afirmam que a precisão e a acurácia das estimativas da severidade aumentam quando a avaliação é realizada com escalas diagramáticas, em comparação com avaliações realizadas sem o auxílio dessas ferramentas.

A utilização da escala diagramática para mancha preta dos citros, com seis níveis para os sintomas do tipo mancha dura e falsa melanose, permitiu quantificar os sintomas da doença em frutos de maneira acurada e precisa. Esses resultados sugerem que o seu uso em estudos epidemiológicos poderá proporcionar informações mais realistas a respeito do patossistema Guignardia citricarpa - citros, assim como, melhores avaliações de diferentes estratégias de controle da mancha preta dos citros.

\section{REFERÊNCIAS BIBLIOGRÁFICAS}

AGUILAR-VILDOSO, C.I., RIBEIRO, J.G.B., FEICHTENBERGER, E., GÓES, A. \& SPÓSITO, M.B. Manual Técnico de Procedimentos da Mancha Preta dos Citros. Brasília. MAPA/DAS/DDIV. 2002.

AMORIM, L. Avaliação de doenças. In: Bergamin Filho, A., Kimati, H. \& Amorim, L. (Eds.). Manual de Fitopatologia v.1. São Paulo. Editora Ceres. 1995. pp.647-671.

CAMPBELL, C.L. \& MADDEN, L.V. Introduction to Plant Disease Epidemiology. New York. John Wiley \& Sons. 1990.

DRAPER, N.R. \& SMITH, H. Applied Regression Analysis. 3. ed. New York. John Wiley \& Sons. 1998.

FEICHTENBERGER, E., MULLER, G.W. \& GUIRADO, N. Doenças dos citros. In: Kimati, H., Amorim, L., Bergamin Filho, A., Camargo, L.E.A. \& Rezende, J.A.M. (Ed.). Manual de Fitopatologia v.2. São Paulo. Editora Ceres. 1997. pp.261-296.

HORSFALL, J.G. \& BARRAT, R.W. An improved grading system for measuring plant diseases. Phytopathology 35:655. 1945. (Abstract)

JORGE, L.A.C. Siarcs 3.0 para Windows: Manual do Usuário. São Carlos. Instrumentação Agropecuária. Embrapa, 1996.

KLOTZ, L.J. Fungal, bacterial and nonparasitic diseases and injuries originating in the seedbed, nursery and orchard. In: Reuther, W., Calavan, E. C. \& Carman, G. E. (Eds.) The Citrus Industry. University of California. v. 4. 1978. pp.1-66.

KOTZÉ, J.M. Black spot. In: Whiteside, J.O., Garnsey, S.M. \& Timmer, L.W. (Eds.). Compendium of Citrus Diseases. Saint Paul. APS Press. 1988. pp.10-12.

KOTZÉ, J.M. Epidemiology and control of citrus black spot in South Africa. Plant Disease 65:945-950. 1981.

KRANZ, J. Measuring plant disease. In: Kranz, J. \& Rotem, J. (Eds.) Experimental Techniques in Plant Disease Epidemiology. Berlin. Springer. 1988. pp.35-50.

NUTTER Jr., F.W. \& SCHULTZ, P.M. Improving the accuracy and precision of disease assessments: selection of methods and use of computer-aided training programs. Canadian Journal of Plant Pathology 17:174-184. 1995.

NUTTER Jr., F.W. \& WORAWITLIKIT, O. Disease.Pro: A computer program for evaluating and improving a person ability to assess disease proportion. Phytopathology 79:1135. 1989.(Abstract)

NUTTER Jr., F.W., MILLER, D.L. \& WEGULO, S.N. Do standard diagrams improve the accuracy and precision of disease assessment?. In: $7^{\text {th }}$ International Congress of Plant Pathology, Edinburgh. v.2. 1998. p.2.1.16.

NUTTER Jr., F.W., TENG, P.S. \& SHOKES, F.M. Disease assessment terms and concepts. Plant Disease 75:1187-1188. 1991.

TENG, P.S. Validation of computer models of plant disease epidemics: a review of philosophy and methodology. Zeitschrift für Pflanzenkrankheiten und Pflanzenschutz 88:455-459. 1981.

TORMELIN, J.R. \& HOWELL, T.A. Distrain: a computer program for training people to estimate disease severity on cereal leaves. Plant Disease 72:455-459. 1988. 